Folgende Erkenntnisse zur privaten Bildschirmmediennutzung können festgehalten werden:

- Insbesondere die Nutzung interaktiver Medien ist in den letzten 10 Jahren stark angestiegen.

- Da sich hinsichtlich der Nutzung des Fernsehens kaum Veränderungen ergeben haben, interaktive Unterhaltungsmedienzeit aber kontinuierlich zugenommen hat, ist insgesamt von einem maßgeblichen Anstieg der Bildschirmmedienzeit auszugehen. Medienaktivitäten binden den größten Zeitanteil im Freizeitmenu von 15-Jährigen.

- Bildschirmmediennutzung unter Heranwachsenden ist primär unterhaltungsmotiviert. Info- und Edutainmentangebote sind im Mediennutzungsmenu insgesamt unterrepräsentiert.

- Sowohl das Fernseh- als auch das Computerspielangebot weist ein hohes Maß an Gewalt und aggressiven Interaktionen auf. Beim Computerspielen ist insbesondere von einem qualitativen Anstieg der Gewalt auszugehen, indem der Gewaltrealismus und die Detailfülle der Darstellungen stark zugenommen haben. Die Präsentation von Gewalt in Computerspielen weist gegenüber dem Fernsehen eine weit höhere Ereignisfrequenz auf.

- Jungen weisen eine auffälligere Mediensozialisation auf, indem sie im Vergleich zu Mädchen früher mit eigenen Mediengeräten ausgestattet sind, höhere Nutzungszeiten aufweisen und häufiger entwicklungsbeeinträchtigende Angebote nutzen.

- Insbesondere hinsichtlich der Unterhaltungsmediennutzung im Kindesalter existieren maßgebliche bildungsmilieuspezifische Unterschiede, die jedoch mit höherem Lebensalter zunehmend nivelliert werden.

\title{
1.2 Bildschirmmediennutzung und Schulleistung
}

In diesem Kapitel wird dargestellt, inwieweit die Medienumwelt von Kindern und Jugendlichen in multifaktoriellen Modellen zur Erklärung von Schulleistungen berücksichtigt wird und welcher Stellenwert ihr dabei im Vergleich zu anderen Faktoren zugeschrieben wird (Kapitel 1.2.1). Daraufhin werden die empirische Forschung (Kapitel 1.2.2) sowie Erklärungshypothesen (Kapitel 1.2.3) zum Zusammenhang von Bildschirmmediennutzung und Schulleistung dargestellt. Der Forschungsstand sowie die Hypothesenlage bilden die Grundlage eines Arbeitsmodells, welches an der (bislang weitestgehend ungeklärten) Frage nach der Art des Zusammenhangs von Bildschirmmediennutzung und Schulleistung anknüpft (Kapitel 1.2.4). Hierauf basiert das Forschungsprojekt Mediennutzung und Schulleis- 
tung des Kriminologischen Forschungsinstituts Niedersachsen (KFN), in welches diese Arbeit funktional eingebettet ist.

\subsubsection{Stellenwert der Medienumwelt in multifaktoriellen Modellen zur Erklärung von Schulleistung}

"Die Schulleistung ist multipel determiniert, d.h. sie hängt in komplexer Weise von individuellen, schulischen und familiären Bedingungsfaktoren gleichermaßen ab. (...) Wie diese verschiedenen Einflußfaktoren bei der Determination der Schulleistungen miteinander zusammenhängen und -wirken, ist jedoch weitgehend unklar" (Helmke \& Weinert, 1997, S. 139).

Wie gezeigt werden konnte, hat sich die Medienumwelt von Kindern und Jugendlichen in den letzten zehn Jahren deutlich verändert. Da das Freizeitleben von Kindern vorrangig durch das Fernsehen bestimmt wird und sich Jugendliche im Alter von 15 Jahren in ihrer Freizeit maßgeblich den drei Aktivitäten Fernsehen, Internetchatten und Computerspielen zuwenden, stellt sich die Frage, inwieweit diesen Verhaltensweisen auch eine Relevanz für die kognitive und schulische Leistungsentwicklung zukommt. In diesem Kapitel soll zunächst betrachtet werden, inwieweit die Medienumwelt von Kindern und Jugendlichen in theoretischen Konzeptionen zur Erklärung von Schulleistung berücksichtigt wird und welcher Stellenwert diesem Faktor dabei zugeschrieben wird.

Im Vergleich zum weiter zu fassenden Schulbildungsbegriff, welcher allgemeine Bildungsziele wie Kompetenzen zum autonomen Handeln, Kompetenzen zu sozialer Partizipation, Lern- und Problemlösekompetenzen und musische, geisteswissenschaftlich-historische, sozialwissenschaftlich-ökonomische und religiöswertbezogene Bildungsziele beinhalten kann (vgl. Weinert, 2002), wird unter Schulleistungen in der Regel im engeren Sinne der Erwerb bereichsspezifischer Kenntnisse, Fertigkeiten und Leistungen verstanden, die im Rahmen schulbezogener Aufgaben und Leistungstests überprüfbar sind (Helmke \& Weinert, 1997). Als zentraler Indikator schulbezogener Leistungen gehören nach Helmke (1997) sowie Helmke und Weinert (1997) aktuelle und kumulative, spezifische und globale Leistungen, die sich aus Tests oder Lehrerbeurteilungen ergeben und in Form von Schulnoten vorliegen. Neuere kompetenzorientierte Ansätze, welche Schulleistungen eher als psychologische Konstrukte operationalisieren, fokussieren in Anlehnung an die Expertiseforschung stärker auf den Erwerb langfristiger Expertise, die sich nicht nur durch den Erwerb bereichsspezifischer Wissensbestände sondern auch die Kompetenz ihrer Anwendung auszeichnet. Kompetenzorientierte Ansätze lösen sich damit von der engeren Auffassung von Schulleistung als curriculare Verfügbarkeit bereichsspezifischen Wissens und betonen die Relevanz 
kognitiver Fähigkeiten und Fertigkeiten zur Lösung spezifischer Probleme (Köller \& Baumert, 2008).

Um interindividuelle Unterschiede in der Schulleistung zu erklären, gehen gängige Konzeptionen im Rahmen multikausaler Erklärungsmodelle von einer Vielzahl determinierender Faktoren aus. Helmke und Weinert (1997) unterscheiden in ihrer bekannten Überblicksarbeit nach einer umfassenden Systematisierung des Forschungsstandes allgemeine Kontextbedingungen (schulexterne, schulinterne und klasseninterne Faktoren), individuelle Determinanten auf Seiten des Schülers (konstitutionelle und kognitive sowie motivationale und affektive Determinanten), familiäre Determinanten (genetische, sozioökonomische) und Determinanten in der Art des Unterrichts und der Lehrerpersönlichkeit. In dieser Konzeption findet sich mit der Kategorie "Andere Sozialisationsinstanzen: Gleichaltrige, Medien" auch die in aktuelleren Modellen aufgegriffene Medienumwelt wieder, so etwa im Kompetenzerwerbsmodell des Programme for International Student Assessment (PISA), welches im Rahmen ihrer Untersuchung die Ausstattung mit Mediengeräten berücksichtigt (Baumert, Artelt, Carstensen, Sibberns, \& Stanat, 2001).

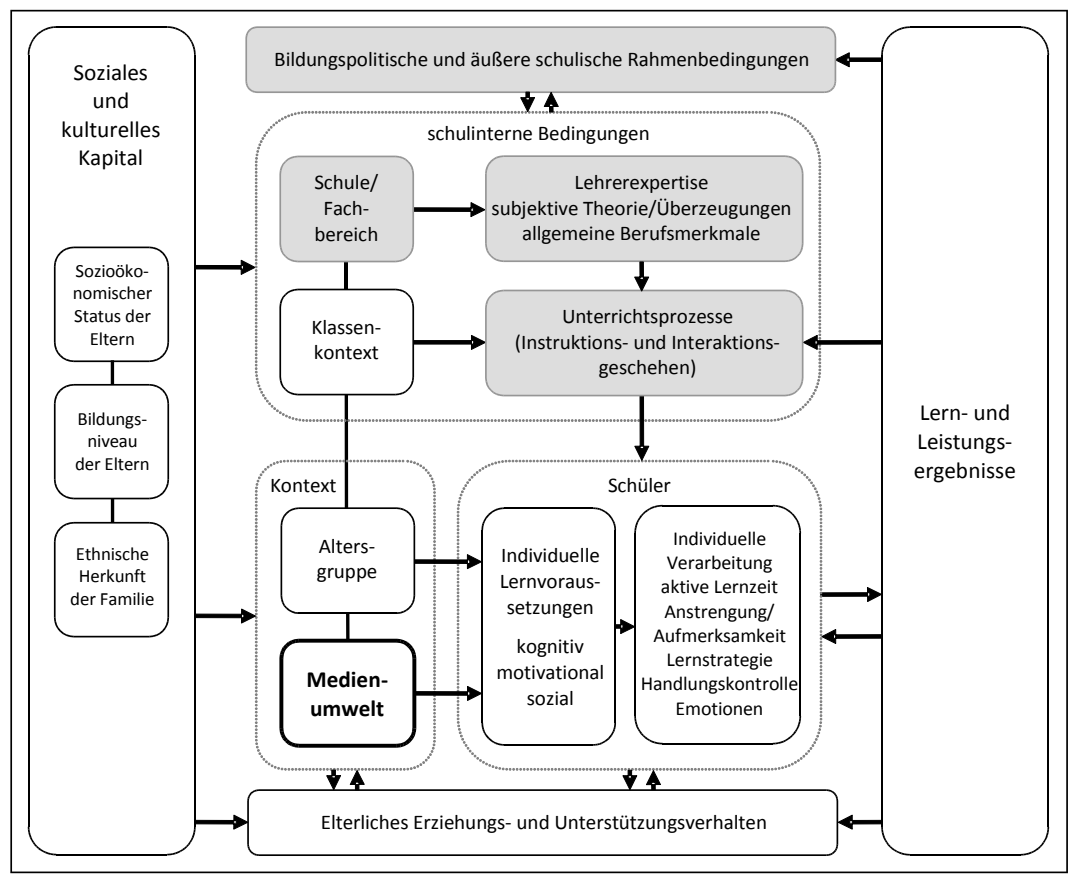

Abbildung 4. Integratives Modell schulischer Leistungsentwicklung (Baumert, et al., 2002; Bos, et al., 2003; Haertel, Walberg, \& Weinstein, 1983; Helmke \& Weinert, 1997; Wang, Haertel, \& Walberg, 1993) 
Zur theoretischen Einbettung des Projektes Mediennutzung und Schulleistung legt unsere Arbeitsgruppe ein integratives Modell der aktuellen nationalen Schulleistungsforschung zugrunde (vgl. Abbildung 4). In diesem sind die Modelle aus dem Programme for International Student Assessment - Pisa (vgl. Baumert, et al., 2002) und der Internationalen Grundschul-Lese-Untersuchung - IGLU (vgl. Bos, et al., 2003) zusammengefasst. Beide Modelle gehen wiederum auf Haertel, Walberg und Weinstein (1983), Wang, Haertel und Walberg (1993) sowie auf Helmke und Weinert (1997) zurück. Das Modell liefert damit einen umfassenden Überblick über die diskutierten Bedingungsfaktoren schulischer Leistungsentwicklung.

Eine übergeordnete Bedeutung für die schulische Leistungsentwicklung wird innerhalb der multifaktoriellen Konzeptionen der Person des Schülers und damit den "konstitutionellen, kognitiven und motivationalen" Lernvoraussetzungen (Helmke, 1997, S. 203) bzw. kognitiven, motivationalen und volitionalen Schülermerkmalen (Schrader \& Helmke, 2008) zugeschrieben (vgl. auch Helmke \& Weinert, 1997, S. 103). Die Annahme, dass kognitive Fähigkeiten für schulische Leistungen von großer Bedeutung sind, konnte in verschiedenen Studien nachgewiesen werden. Allgemeine Intelligenz und Schulleistungen korrelieren im Mittel mit $r=.50$ (für einen Überblick vgl. Helmke \& Weinert, 1997; Köller \& Baumert, 2008, S. 759). In der nationalen Ergänzung der PISA-Studie 2003 in Deutschland wurde die kognitive Grundfähigkeit der Schüler mit dem Untertest Figurenanalogien (N2) des Kognitiven Fähigkeits-Test (KFT 5-12+R) operationalisiert (Ramm, et al., 2006). ${ }^{16}$ Der KFT ist ein differenzieller Intelligenztest bestehend aus neun Untertests und beansprucht eine Erfassung des kognitiven Gesamtleistungsniveaus von Schülern (Heller \& Perleth, 2000). Die Ergebnisse des Untertests weisen mittlere bis hohe Korrelationen mit Schulleistungen in den Fächern Mathematik ( $r=$. $74)$, Lesen $(r=.64)$ und Naturwissenschaften $(r=.68)$ auf. Kognitive Leistungsfähigkeit und schulisches Lernen bedingen sich jedoch wechselseitig. Studien konnten zeigen, dass die Intelligenzentwicklung auch von der besuchten Schulform und den erworbenen Kompetenzen mit bestimmt wird (vgl. Helmke \& Weinert, 1997). Somit wird davon ausgegangen, dass das Lernen zum einen über neue Wissensbestände die kristalline Intelligenz erhöht, zum anderen eine Strukturierung bereits vorhandener Umwelterfahrungen fördert, indem diese wissenslogisch im Sinne der einzelnen Schulfächer angeordnet und kategorisiert werden und damit eine Dekontextualisierung des Wissens angeregt und semantische Gedächtnisinhalte gefördert werden (Oerter \& Montada, 2008, S. 255).

16 Der Untertest Figurenanalogien beansprucht die Erfassung nonverbaler, figural-räumlicher Fähigkeiten. Vorgegeben ist ein Figurenpaar, welches ein bestimmtes Verhältnis repräsentiert. $\mathrm{Zu}$ einer dritten Figur muss aus einer Auswahl von fünf weiteren Figuren die Figur identifiziert werden, die mit der dritten Figur im selben Verhältnis steht wie die beiden Figuren des vorgegebenen Paares (Heller \& Perleth, 2000). 
Die Annahme einer besonderen Bedeutung der kognitiven Lernvoraussetzungen widerspricht dabei nicht der Annahme, dass schulische Leistungen multifaktoriell bedingt sind (vgl. Heller, 1997), sondern folgt vielmehr der Auffassung, dass schulische Leistungen als Ergebnis einer Reihe von den Lernerfolg nur indirekt und einer Reihe von den Lernerfolg direkt beeinflussenden Faktoren verstanden werden können (Schrader \& Helmke, 2008). Dabei wird in der Regel davon ausgegangen, dass sich die vielfältigen Einflussfaktoren je nach Vorzeichen additiv verstärken oder kompensatorisch abschwächen und innerhalb dieses wechselseitigen Bedingungsgefüges auf die schulischen Leistungen einwirken und sie bestimmen (vgl. Helmke \& Weinert, 1997, S. 139). In den vorhandenen Konzeptionen zur multikausalen Erklärung von Schulleistungen wird der Medienumwelt wie den meisten anderen Kontextfaktoren keine vorrangige Bedeutung für die schulische Leistungsentwicklung beigemessen (Schrader \& Helmke, 2008). Das Begriffsverständnis von "Medienumwelt" erscheint dabei jedoch häufig sehr weit gefasst, indem darunter sowohl die schulische als auch die private Ausstattung des Schülers mit Mediengeräten, die zeitliche Beschäftigungsdauer sowie die Nutzung spezifischer förderlicher oder problematischer Inhalte oder Formate verstanden wird. So beziehen sich Schrader und Helmke (2008) in einer Diskussion der Determinanten von Schulleistung hinsichtlich des Einflussfaktors Medien auf private Fernsehnutzung, die je nach Art und Intensität sowohl positive wie negative Folgen für die schulische Leistungsentwicklung haben könne und auf den Einsatz von Computern an Schulen, die als Chance zur Optimierung des Lernens zu verstehen sei. Als Medienumwelt wäre demnach sowohl die private Mediennutzung - die wie gezeigt werden konnte hauptsächlich unterhaltungsmotiviert ist - als auch der schulische Einsatz von Medien zur Vermittlung von Lehrinhalten zu verstehen.

Unter Rückgriff auf die Ausführungen in Kapitel 1.1 soll der Begriff Medienumwelt im Folgenden näher eingegrenzt werden, indem dieser als private Mediennutzung von Kindern und Jugendlichen verstanden wird. Diese Definition präzisiert das Verständnis von Medien als Umweltfaktor, da ein Einsatz von Medien im Schulunterricht zur Wissensvermittlung, zur Vermittlung von Lernstrategien und zur Realisierung bestimmter Lernumgebungen (Schrader \& Helmke, 2008) nach dem Modell von Helmke und Weinert (1997) den Prozessmerkmalen des Unterrichts (Unterrichtsprozessen) oder mitunter auch der Lehrer-Schüler-Interaktion zuzuordnen wäre. Die Einordnung der Mediennutzung als schulleistungsdeterminierender Faktor unter den sonstigen Sozialisationsfaktoren lässt hingegen eine Eingrenzung dieses Begriffs auf die private Mediennutzung und Ausstattung von Kindern und Jugendlichen sinnvoll erscheinen (Helmke \& Weinert, 1997). Dies entspricht auch den konzeptionellen Grundlagen der PISA-Studie, in welcher 
Medienumwelt als Teil des Sozialisationskontextes der Altersgruppe aufgefasst wird (Baumert, et al., 2001). ${ }^{17}$

Unter Rückgriff auf das eingeführte Prozessmodell (vgl. Abbildung 4) wird der Medienumwelt innerhalb dieser Modellannahme damit in ähnlicher Weise wie den Unterrichtsprozessen ein möglicher Einfluss auf die individuellen Lernvoraussetzungen und die individuelle Lernverarbeitung des Schülers zugesprochen, die wiederum als maßgeblich für die Lern- und Leistungsergebnisse in der Schule gelten. Die Medienumwelt wird damit, folgt man den Ausführungen von Schrader und Helmke (2008), nicht als proximaler, die Lernleistung unmittelbar bestimmender Faktor sondern als distaler, die Lernleistung indirekt beeinflussender Faktor gewertet (vgl. auch Wittwer \& Senkbeil, 2008, S. 1559). An diese Überlegungen schließt sich unmittelbar die Frage an, in welcher Weise und vermittelt über welche Mediatoren diesem Faktor Bedeutung für schulische Leistungen zukommen könnte.

Bevor diese Frage erneut aufgegriffen wird, soll im Folgenden zunächst anhand des verfügbaren Forschungsstandes überprüft werden, inwieweit Schulleistungen mit dem privaten Mediennutzungsmenu von Kindern und Jugendlichen überhaupt variieren. Dabei soll primär geklärt werden, ob die im Zentrum der Betrachtung stehenden privaten Mediennutzungsparameter Nutzungsdauer und Nutzungsinhalte schulische Leistungen positiv oder negativ beeinflussen können. Die Ausführungen dieses Kapitels machen jedoch bereits deutlich, dass aufgrund des multifaktoriellen Zustandekommens von Schulleistungen (Helmke \& Weinert, 1997), der Komplexität und Breite des Konstrukts, der distalen Position der Medienumwelt des Schülers innerhalb des vermuteten Wirkgefüges (Schrader \& Helmke, 2008) sowie der grundsätzlichen Annahme möglicher wechselseitiger Kompensationen zwischen den bedingenden Faktoren (Helmke \& Weinert, 1997) nur geringe Zusammenhänge zwischen beiden Phänomenen erwartet werden können.

\subsubsection{Forschungsstand: Bildschirmmediennutzung und Schulleistung}

Im nachfolgenden Überblick werden Studien ausgeklammert, die ausschließlich einer mediengerätegeleiteten Perspektive folgen und nur nach der Geräteausstattung bzw. der Nutzung oder Nichtnutzung bestimmter Bildschirmgeräte differen-

17 Natürlich kann an dieser Stelle eingewendet werden, dass die private Medienumwelt längst Einzug in den Schulalltag gehalten hat, indem Handys zum Abspielen von Videos und Videospielen sowie mobile Spielkonsolen auch in die Schule mitgenommen werden bzw. schulisches Medienequipment auch privat von den Schülern genutzt wird. Die Eingrenzung betrifft also weniger den Ort der Nutzung als vielmehr die Abgrenzung privater Mediennutzung von einem professionellen und pädagogisch angeleiteten Einsatz von Medien im Rahmen des Schulunterrichts oder zur Verfolgung bestimmter pädagogischer Ziele. 
zieren. Ein solcher Auflösungsgrad erscheint für die vorliegende Arbeit zu unpräzise. Dies wird gerade am Beispiel des Computers deutlich, der als sog. Konvergenzmedium ein breites Nutzungsspektrum von der "E-Mail-Korrespondenz über die Nutzung von Textverarbeitungs-, Lernprogrammen und Computerspielen bis hin zur Bearbeitung von Fotos und eigenen Programmiertätigkeiten bereit hält, so dass hier die (...) Annahme medienspezifischer Wirkungsannahmen geradezu naiv erscheinen (muss)" (Ennemoser, 2007, S. 198). Aber auch die Unterscheidung von Spielkonsolennutzern und -nichtnutzern sowie Fernsehnutzern und -nichtnutzern lässt viele Fragen hinsichtlich der präferierten Angebote sowie der täglichen Nutzungsdauer offen. Die Bedeutung heimischer Computerausstattung bzw. die Frage, inwieweit bereits die bloße Nutzung des Computers Schulleistungen positiv oder negativ beeinflusst, wird so auch kaum überraschend kontrovers und für die Debatte wenig gewinnbringend diskutiert. ${ }^{18}$

Um die Historie der Erforschung des Zusammenhangs von Bildschirmmediennutzung und Schulleistung und die hiermit in Zusammenhang stehenden Erklärungshypothesen zu veranschaulichen wird zunächst auf die Fernsehnutzung eingegangen. Da in dieser Arbeit jedoch ein Fokus auf die kognitiven Wirkungen gewalthaltiger Computerspiele gelegt wird, soll ein Schwerpunkt auf die Studienlage zum Zusammenhang von Computerspielnutzung und Schulleistung gelegt werden. Aufgrund des Bedeutungsgewinns interaktiver Bildschirmmedien in den letzten zehn Jahren und der qualitativen Intensivierung der Gewalt in gerade diesem Medium (vgl. Kapitel 1.1.2) konzentriert sich der Forschungsüberblick auf Veröffentlichungen aus dem Zeitraum 1999 bis 2008. Studien wurden dem Gegenstandsbereich zugeordnet, wenn als abhängige Variablen schulische Leistungen in Form von Noten oder Prüfungsleistungen oder schulische Leistungsfähigkeit im Sinne von Schulleistungs- oder Grundkompetenztestergebnisse (z. B. Lesefähig-

18 Ein Beispiel hierfür lässt sich der Debatte um die PISA Befunde entnehmen. So wies die PISA-Studie 2003 für alle OECD-Länder schlechtere Leistungen in Mathematik für Schüler aus, die keinen heimischen Zugang zu einem Computer besitzen (OECD, 2006). Nach Kontrolle des sozioökonomischen Status im Elternhaus wird diese Differenz immer noch für 23 der 31 OECD-Staaten signifikant und fällt in 7 OECD Staaten, darunter Deutschland, mit mehr als 30 Punkte (entsprechend einem halben PISA-Kompetenzlevel) am größten aus. In ähnlicher Weise fallen die Mathematikleistungen auch bei jenen Schülern schlechter aus, die Computer selten oder gar nicht nutzen. Wittwer und Senkbeil (2008) führten eine Re-Analyse der PISA-Daten durch und berücksichtigen dabei die Variablen Geschlecht, Migrationshintergrund, sozioökonomischer Status, kognitive Grundfähigkeit (gemessen mit dem Untertest Figurale Analogien des Kognitiven Fähigkeitstests) und die Nutzung anderer Medien wie Lesen und Fernsehen. Werden diese Variablen im Rahmen von Regressionsanalysen berücksichtigt, verliert der von der OECD als positiv berichtete Zusammenhang (OECD, 2006) zwischen der heimischen Verfügbarkeit eines Computers und besseren Mathematiknoten seine statistische Bedeutung. Der gesamten Debatte lässt sich dabei nicht entnehmen, wie intensiv das Medium tagtäglich eingesetzt wird und welche Inhalte dabei genutzt werden. Ein ähnlicher Widerstreit mit ähnlichem Ausgang ergab sich bereits in der Replik zur PISAStudie 2001, vgl. Fuchs und Wößmann (2004; für einen Überblick siehe Mößle, et al., 2007; OECD, 2001). 
keit) berücksichtigt werden. ${ }^{19}$ Unter diesem Fokus wurden im Mai 2008 und im April 2009 den bereits im Rahmen der Projektarbeit vorliegenden Forschungsstand aktualisierende Literaturrecherchen in den Datenbanken PubMed, PsyCONTENT und Google Scholar durchgeführt. ${ }^{20}$

\subsubsection{Fernsehnutzung und Schulleistung}

Während in den 1950er und bis in die 1970er Jahre hinein noch mehrheitlich davon ausgegangen wurde, dass Fernsehnutzung und Schulleistung annähernd unkorreliert seien (vgl. Gaddy, 1986), überwiegen seit den 1980er Jahren Studien, die übergreifend einen negativen Zusammenhang ${ }^{21}$ zwischen beiden Variablen nahelegen (vgl. Ennemoser \& Schneider, 2007). So konstatieren Williams, Haertel, Haertel und Walberg (1982) auf Basis einer Metaanalyse von insgesamt 23 internationalen Studien des Veröffentlichungszeitraums 1954 bis 1980 einen negativen - wenngleich mit $r=-.05$ sehr geringen - Zusammenhang zwischen Fernsehnutzung und Schulleistung. In einer Erweiterung dieser Analyse von Neuman (1988) um US-Studien der Jahre 1978 bis 1983 zeigt sich unter Kontrolle des sozioökonomischen Status im Elternhaus ein Zusammenhang zwischen einer erhöhten Fernsehzeit und geringeren Lesefähigkeiten (Vocabulary, Comprehension, Study Skills). Comstock und Scharrer (1999) legen in ihrem Literaturüberblick einen Schwerpunkt auf das California Assessment Program (CAP), in welchem in den 1980er Jahren Lese-, Rechen- und Schreibleistungen sowie Fernsehnutzungszeiten von 282.000 Schülern sechster und 227.000 Schülern zwölfter Klassen erfasst wurden. Hinsichtlich aller drei erfassten Leistungsbereiche und über alle sozioökonomischen Statuslevel hinweg fanden sich negative Zusammenhänge mit der Fernsehzeit. Die negative Beziehung fällt stärker für 12.-Klässler als für 6.-Klässler sowie für Kinder mit höherem als geringerem sozioökonomischen Status aus (Comstock \& Scharrer, 1999). In seiner Metaanalyse von Studien der späten 1980er bis späten 1990er Jahre mit mehr als einer Millionen Schülern ermittelt Razel

19 Die Frage nach dem Zusammenhang zwischen Bildschirmmediennutzung und kognitiven Teilleistungsaspekten wird im Kapitel 1.3.2 dieser Arbeit am Beispiel der Computerspiele aufgegriffen.

20 Es wurden deutsche und englische Suchbegriffe eingesetzt. Auf AV-Seite waren dies schulleistung, school achievement, school performance, scholastik achievement, educational achievement und educational performance, hinsichtlich der UV-Ebene fernsehen, tv, television, computerspiel, videospiel, computer game, video game. Zusätzlich wurden Termini wie langzeitstudie, längsschnittstudie, longitudinal analysis, longitudinal study, metaanalyse und meta analysis eingesetzt, um besonders einschlägige Studien zu identifizieren. Zusätzlich wurden im "Schneeballverfahren" Studien anhand vorhandener Quellennachweise einbezogen.

21 Im Folgenden wird immer dann von einem "negativen Zusammenhang" gesprochen, wenn die Mediennutzung mit schlechteren Leistungen bzw. schlechteren Noten in Beziehung steht. 
(2001) 305 Korrelationen zwischen Fernsehnutzung und Schulleistung. 90 Prozent der Korrelation erweisen sich als negativ. Der Autor fasst die Befunde - auch unter Rückgriff auf die Studien von Williams et al. (Williams, et al., 1982) und Neuman (1988) - wie folgt zusammen: "Those results leave little room for doubt concerning the negative nature of the overall linear relationship between television viewing and educational achievement" (Razel, 2001, S. 372).

In zwei älteren Panelstudien konnte jedoch der postulierte negative Zusammenhang zwischen Fernsehnutzung und Schulleistung bei Kontrolle von Intelligenz und sozioökonomischem Status nicht bestätigt werden (Gaddy, 1986; Gortmaker, Salter, Walker, \& Dietz, 1990). Die Studie von Gortmaker et al. (1990) basierte auf einem Kinderpanel, dessen beide Erhebungswellen in den frühen und späten 1960er Jahren durchgeführt wurden, die Studie von Gaddy (1986) auf einem Panel von Collegestudenten aus den frühen 1980er Jahren. Beide Studien waren in der Folge ihrer Veröffentlichung einiger Kritik ausgesetzt, die sich insbesondere auf den methodischen Umgang der Autoren mit Kontrollvariablen, die Maße zur Erfassung der Fernsehnutzungszeit und die mangelnde Berücksichtigung der genutzten Fernsehinhalte bezog. ${ }^{22}$ Abweichend von den Befunden von Gaddy (1986) und Gortmaker et al. (1990) ergab sich in einer aktuelleren Längsschnittstudie aus den neunziger Jahren in einem Panel von $\mathrm{N}=1.050$ Grundschülern ein negativer $\mathrm{Zu}$ sammenhang zwischen Fernsehzeit und dem Leseverständnis im Folgejahr und dies in gleicher Weise für drei unterschiedliche sozioökonomische Statuslevel sowie für Kinder mit geringer, mittlerer und hoher Intelligenz. Je nach genutzten Inhalten zeigten sich gegenläufige Befundmuster, indem Unterhaltungsfernsehzeit negativ, Edutainmentfernsehzeit positiv, jedoch nicht signifikant, mit Leseverständnis im Folgejahr korreliert (Koolstra, van der Voort, \& van der Kamp, 1997).

Aufgrund der sich widersprechenden Befundlage längsschnittlicher Daten bis in die späten 1990er Jahre hinein galt die Evidenz für einen negativen kausalen Zusammenhang lange Zeit noch als schwach. Mit den Studien der letzten zehn Jahre (vgl. Anhang A) fand diese Annahme jedoch in internationalen Studien vom Kindes- bis ins Jugendalter sowie in Querschnitts- und Längsschnittstudien vermehrt Bestätigung und hat sich in der Folge zunehmend durchgesetzt. So zeigten sich in zwei US-amerikanischen (J. G. Johnson, Cohen, Kasen, \& Brook, 2007; Zimmerman \& Christakis, 2005), einer neuseeländischen (Hancox, Milne, \& Poul-

22 So wurde beispielsweise angeführt, dass in beiden Studien nicht nur obligatorische Variablen wie der sozioökonomische Status und die Intelligenz kontrolliert wurden, sondern eine Vielzahl weiterer Merkmale wie etwa die aktuelle TV-Nutzung, frühe Leistungstestergebnisse, Jahreszeit, Region, Bevölkerungsdichte, Alter der Eltern, Kinderzahl und ethnischer Hintergrund, wodurch ein möglicherweise vorliegender Effekt des Fernsehens nur noch schwer aufzudecken sein könnte (Comstock \& Scharrer, 1999, S. 251 ff.). Hinsichtlich beider Studien wurde zudem die Reliabilität der angewendeten Abfragemethode der Fernsehzeit in Frage gestellt (Beentjes \& Van der Voort, 1988, S. 405). 
ton, 2005) und einer deutschen (Ennemoser \& Schneider, 2007) Längsschnittstudie auch unter Kontrolle des sozioökonomischen Status und kognitiver Fähigkeiten oder Intelligenz ein negativer Zusammenhang zwischen kindlicher Fernsehnutzung und späteren Schulleistungsparametern. In der neuseeländischen Studie von Hancox und Kollegen zeigte sich, dass erhöhte Fernsehzeiten im Kindes- und Jugendalter die Wahrscheinlichkeit eines späteren Schulabbruchs erhöhen und die Wahrscheinlichkeit für einen späteren Universitätsabschluss vermindern (Hancox, et al., 2005). Zimmermann und Christakis (2005) konnten zeigen, dass jede zusätzliche Stunde, die drei- bis fünfjährige Kinder mit dem Fernsehen verbringen, geringere Lese- und Rechenleistung im Alter von sechs bis sieben Jahren vorhersagt. Johnson und Kollegen konnten ebenfalls aufzeigen, wie eine hohe Fernsehnutzung von mehr als drei Stunden noch im Jugendalter (14 Jahre) negativen Einfluss auf eine Vielzahl von Leistungsindikatoren im Alter von 16, 22 und 33 Jahren nehmen kann. So erhöhte sich mit der Vielnutzung die Wahrscheinlichkeit für schlechtere Noten, Schulversagen und Schulabbruch, während gleichzeitig die Wahrscheinlichkeit für den Besuch einer höheren Schulform verringert wurde (J. G. Johnson, et al., 2007). In der Längsschnittstudie von Ennemoser und Schneider (2007) konnte die zentrale Bedeutung der Unterhaltungsmediennutzung herausgestellt werden, indem unter Kontrolle von Intelligenz und sozioökonomischem Status nur diese negativ mit späterer Leseleistung korrelierte (vgl. Abbildung 5). 


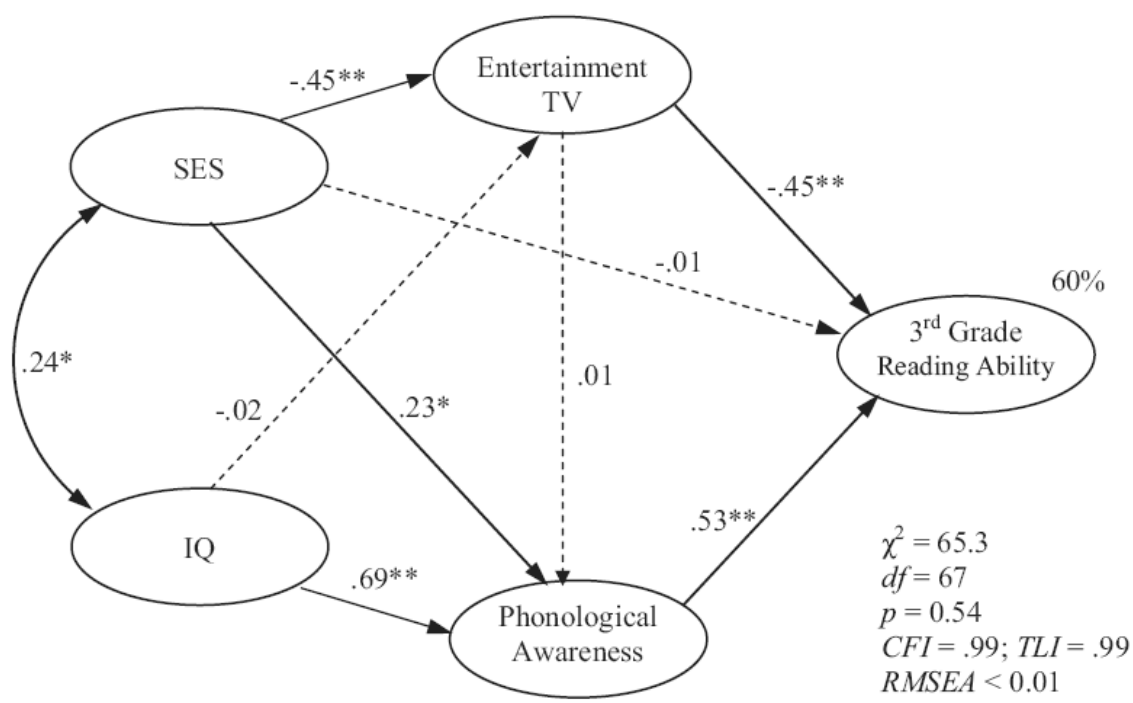

Abbildung 5. Pfadmodell zum Einfluss der Unterhaltungsfernsehnutzung im Kindergartenalter auf die Lesefähigkeit in der dritten Klasse unter Berücksichtigung von sozioökonomischen Statuts (SES), Intelligenz (IQ) und kognitiven Grundvoraussetzungen des Leselernens im Kindergartenalter (Nachdruck aus Ennemoser \& Schneider, 2007, S. 361, mit Genehmigung der American Psychological Association).

Gemischte Befunde ergab eine weitere Längsschnittstudie aus den USA (D. R. Anderson, Huston, Schmitt, Linebarger, \& Wright, 2001). Im Längsschnitt erwies sich eine stärkere Nutzung von Informationsprogrammen im Vorschulalter für Jungen als relevanter Prädiktor besserer Schulleistungen im Jugendalter. Die Nutzung von gewalthaltigen Programmen im Vorschulalter war hingegen mit schlechteren Schulleistungen im Jugendalter verknüpft, dies jedoch nur bei Mädchen (D. R. Anderson, et al., 2001). Drei aktuellere Querschnittsuntersuchungen ermittelten ebenfalls negative Zusammenhänge zwischen Fernsehnutzung und Schulleistung (Gentile, et al., 2004; Mößle, et al., 2007; Shin, 2004). In der Untersuchung von Mößle und Kollegen bleibt der Befund auch dann stabil, wenn dabei Geschlecht, sozioökonomische Variablen und das elterliche Erziehungsverhalten kontrolliert werden. In einem Pfadmodell (vgl. Abbildung 6) zeigt sich ein stärkerer Zusammenhang zwischen den genutzten Medieninhalten und Schulleistung als zwischen der reinen Medienzeit und Schulleistung (Mößle, et al., 2007). 


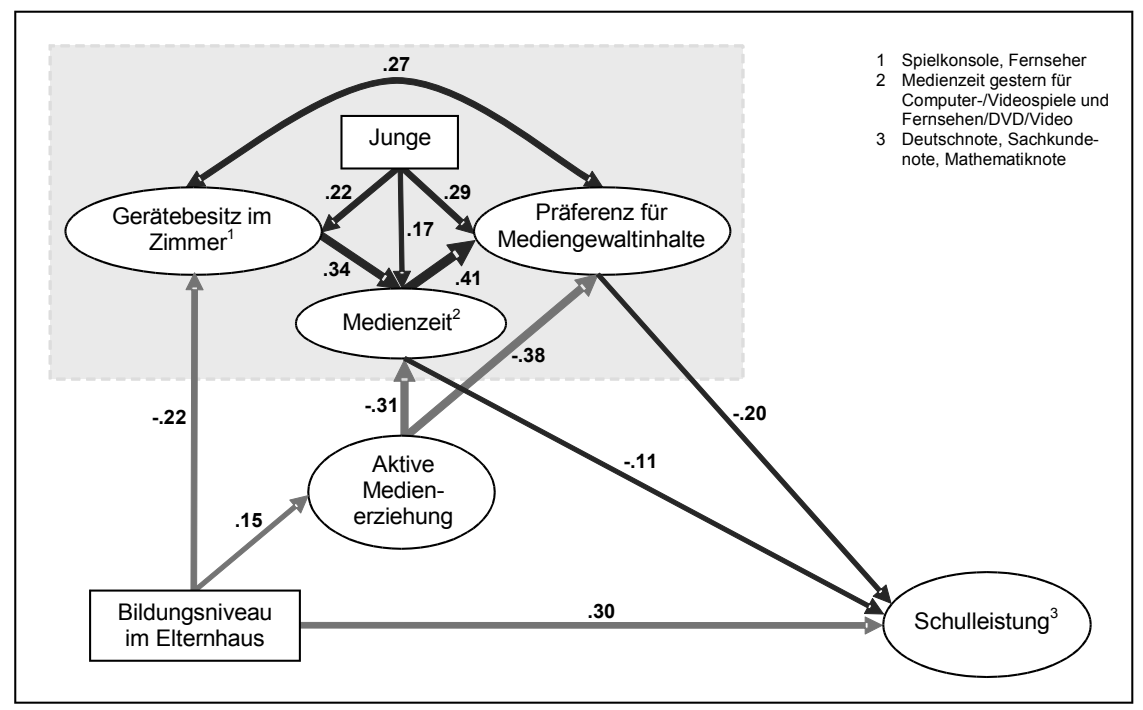

Abbildung 6. Pfadmodell zur Bedeutung von Mediennutzung für die Erklärung von Schulleistung (vgl. Mößle, et al., 2007). Pfade zum gewaltfreien Familienklima und der Sprachfähigkeit bei Einschulung werden aus Gründen der Übersichtlichkeit nicht dargestellt.

Als umstritten gilt nachwievor die Frage, ob es sich bei dem Zusammenhang von Fernsehnutzung und Schulleistung um einen kurvilinearen Zusammenhang handeln könnte. Williams (1982) stellte fest, dass eine Nutzungszeit von wöchentlich bis zu zehn Stunden noch mit besseren Leistungen in Beziehung steht, sich dieser Zusammenhang jedoch ab einer Fernsehdauer von mehr als zehn Stunden pro Woche umkehrt und fortan mit zunehmend schlechteren Schulleistungen einher geht (Williams, et al., 1982). Neumanns Review deutet zwar ebenfalls auf einen kurvilinearen Zusammenhang hin, dies jedoch nur für Schüler der ersten bis fünften (elementary) und der sechsten bis neunten Jahrgangsstufe (intermediate). Hier zeigen sich jedoch erst ab einer Nutzung von wöchentlich mehr als 20 Stunden verringerte Leistungen. Bei Schülern des zehnten bis zwölften Jahrgangs stellt sich der Zusammenhang hingegen als linear dar, indem höhere Nutzungszeiten generell mit schlechteren Leistungen einhergehen (Neuman, 1988). Auch die Metaanalyse von Razel (2001) bestätigt sowohl die Vermutung eines kurvilinearen Zusammenhangs, als auch die Altersabhängigkeit dieser Beziehung und konkretisiert den Befund dahingehend, dass mit zunehmendem Lebensalter eine immer geringere Fernsehnutzung als optimal für schulische Leistungen gelten kann (vgl. Abbildung 7). 
Von einigen Autoren wird jedoch die Relevanz eines möglicherweise vorhandenen kurvilinearen Zusammenhangs angezweifelt (vgl. Comstock \& Scharrer, 1999, S. 248). In der aktuellen Längsschnittstudie von Ennemoser und Schneider findet sich darüber hinaus kein Hinweis auf den postulierten kurvilinearen Zusammenhang (2007). Die Autoren vermuten, dass diese Diskrepanzen auf Mängel in der Erfassung der Fernsehmenus in bisherigen Studien zurückzuführen sein könnte (Ennemoser \& Schneider, 2007, S. 364). Als zusätzliche Erklärung bietet sich an, dass geringere Medienzeiten häufiger mit der Auswahl hochwertiger Programme, höhere Medienzeiten häufiger mit der Auswahl von Unterhaltungsangeboten einhergehen (Comstock \& Scharrer, 1999, S 254). Somit könnte der kurvilineare Zusammenhang auch dadurch zu erklären sein, dass Kinder die keinen Zugang zum Fernsehen haben gegenüber solchen Kindern benachteiligt sind, die geringe Medienzeiten und einen damit typischerweise einhergehenden hohen Anteil von stimulierenden Edutainmentformaten aufweisen. Mit zunehmenden Nutzungszeiten könnten jedoch wiederum - nicht nur aufgrund einer einseitigeren Ausrichtung des Freizeitverhaltens sondern auch aufgrund der stärkeren Dominanz von Unterhaltungsangeboten - nachteilige Wirkungen eintreten:

"Thus, light viewing by young persons is associated with more instrumental and information-oriented use that is unlikely to interfere with and might enhance scholastic performance, whereas heavy viewing reflects not only greater use but greater emphasis on less scholastically useful content" (Comstock \& Scharrer, 1999, S. 254).

Da Ennemoser und Schneider die reine Unterhaltungsfernsehzeit in ihrem Nutzergruppenvergleich betrachten, erscheint es plausibel, dass ihre Daten entgegen der Befunde anderer Studien auf einen linearen Zusammenhang hindeuten und Mittelseher von Unterhaltungsformaten mit einer täglichen Fernsehzeit im Vorschulalter von rund 30 Minuten bereits gegenüber Wenigsehern mit 6 Minuten täglicher Fernsehzeit benachteiligt sind. In ähnlicher Weise könnte der Befund von Razel (2001), dass mit höherem Lebensalter geringere Fernsehzeiten als "ideal" für die schulische Leistungsentwicklung zu bewerten seien, widerspiegeln, dass mit dem Übergang vom Kindes- ins Jugendalter der Anteil von Edutainmentnutzung gemeinhin zurückgeht (vgl. J. G. Johnson, et al., 2007). Somit erscheinen weitere empirische Untersuchungen notwendig, um die funktionale Verbindung zwischen Fernsehnutzung und Schulleistung näher aufzuklären. 
optimale Sehdauer in Abhängigkeit vom Lebensalter

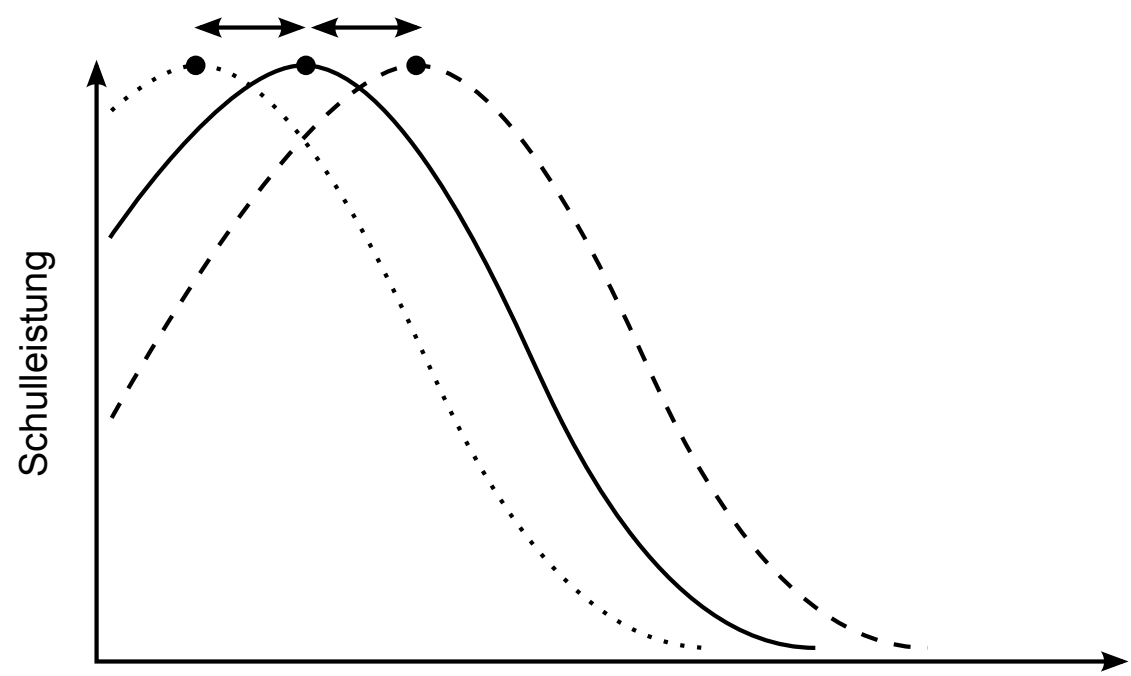

Tägliche Fernsehnutzungszeit

Abbildung 7. Veranschaulichung der Modellannahme eines kurvilinearen $\mathrm{Zu}-$ sammenhangs zwischen Fernsehnutzung und Schulleistung in Abhängigkeit vom Lebensalter (in Anlehnung an Williams et al., 1982; Neuman, 1988; Razel, 2001)

Neben der Frage nach der Art des Zusammenhangs, der angesichts der genannten Befunde weiterhin als umstritten gelten muss (Ennemoser \& Schneider, 2007), sind einige methodische Defizite in den Untersuchungen zu konstatieren. Die meisten Untersuchungen, so auch die aktuelleren Studien der letzten zehn Jahre, konzentrieren sich ausschließlich auf Fernsehnutzungszeiten und berücksichtigen dabei nicht die genutzten Inhalte (Gentile, et al., 2004; Hancox, et al., 2005; J. G. Johnson, et al., 2007; Sharif \& Sargent, 2006; Zimmerman \& Christakis, 2005). Unter den drei aktuelleren Studien, die Inhalte der Fernsehnutzung berücksichtigt haben, wird nur in zwei Arbeiten detailliert nach der Nutzung mutmaßlich entwicklungsbeeinträchtigender und stimulierender Formate unterschieden (D. R. Anderson, et al., 2001; Mößle, et al., 2007). In der Untersuchung von Ennemoser und Schneider (2007) findet hingegen eine Dichotomisierung der genutzten Formate nach Unterhaltungs- und Edutainmentfernsehen statt (Ennemoser \& Schneider, 2007). In allen drei Untersuchungen erweisen sich jedoch die Nutzungsinhalte als bedeutsam für den Zusammenhang mit Schulleistungen, indem sich gerade die Nutzung von Entertainmentangeboten (Ennemoser \& Schneider, 2007) und die 
Nutzung gewalthaltiger Angebote (D. R. Anderson, et al., 2001; Mößle, et al., 2007) als maßgeblich für den negativen Zusammenhang zwischen Fernsehnutzung und Schulleistung herausstellen. Dies weist umso dringender auf die Notwendigkeit hin, in Studien zur Bildschirmmediennutzung und Schulleistung die genutzten Inhalte zu berücksichtigen. Weitere Kritik bezieht sich auf die Abfragemethoden der Fernsehnutzung, die deutlich zwischen den Studien variieren und in Teilen auch nicht zufriedenstellend gelöst ist (vgl. die Ausführungen von Ennemoser \& Schneider, 2007). Zudem sind einige Studien von zweifelhafter Aussagekraft, indem sie gravierende Defizite hinsichtlich der Kontrolle relevanter Drittvariablen aufweisen (vgl. D. R. Anderson, et al., 2001; Gentile, et al., 2004; Sharif \& Sargent, 2006; Shin, 2004).

\subsubsection{Computerspielnutzung und Schulleistung}

Bis in die späten 1990er Jahre hinein existierten nur wenige Studien zu der Frage, ob auch Computerspielnutzung mit schulischen Leistungen in Zusammenhang steht. Mitte der 1980'er Jahre, als Videospiele noch am Anfang ihrer Entwicklung standen und die Verbreitung weitaus geringer war als heute, berichten Harris und Williams (1985) im Rahmen einer kleinen Querschnittsuntersuchung von N = 152 High-School-Schülern negative Zusammenhänge zwischen der Spielzeit und der Note in Englisch $(r=-.28)$ sowie zwischen dem für Videospiele ausgegebenen Geld und der Note in Englisch $(r=-.20)$. Zwei Jahre später erschien die Studie von Lin und Lepper (1987), mit der mögliche Zusammenhänge zwischen verschiedenen Freizeitbeschäftigungen und Schulleistung untersucht werden sollten. Hierbei zeigten sich bei Jungen negative Zusammenhänge zwischen der Nutzung von Arcadeautomaten und Lehrerurteilen zu mathematischen Fähigkeiten sowie genereller Schulleistungsfähigkeit. Der Heimgebrauch von Videospielen stand hingegen nicht mit Schulleistungen in Zusammenhang (Lin \& Lepper, 1987). Van Schie und Lippert fanden in einer niederländischen Stichprobe aus dem Jahr 1994 von insgesamt 346 Schülern 7. und 8. Klassen $(M[$ Alter] = 11.5) die in sieben Schulen rekrutiert wurden, ebenfalls keinen Zusammenhang zwischen dem Heimgebrauch von Videospielen und Schulleistung, selbst unter Berücksichtigung der verbalen und nonverbalen Intelligenz der Schüler (van Schie \& Wiegman, 1997).

Aufgrund der rasanten technischen Weiterentwicklung (vgl. Kapitel 1.1.2) und des allgemeinen Bedeutungsgewinns interaktiver Unterhaltungsmedien (vgl. Kapitel 1.1.1) sollten jedoch insbesondere bei Computerspielen aktuellere Studien herangezogen werden. Insgesamt konnten für den infrage stehenden Zeitraum von 1999 bis 2008 neun Querschnittsuntersuchungen aufgefunden werden, von denen 
eine Studie aus Qualitätsgründen ${ }^{23}$ ausgeschlossen werden musste (vgl. Anhang B). Längsschnittstudien konnten nicht aufgefunden werden. Alle acht verbleibenden Studien weisen auf einen negativen Zusammenhang zwischen der mit Computerspielen verbrachten Zeit und Schulleistungen hin, drei davon beziehen sich auf Kinder im Alter zwischen 10 und 13 Jahren (Mößle, et al., 2007; Roberts, Foehr, \& Rideout, 2005; Sharif \& Sargent, 2006), zwei auf Jugendliche im Alter von 14 bis 15 Jahren (Chan \& Rabinowitz, 2006; Gentile, et al., 2004) und drei auf ältere überwiegend erwachsene Populationen (Anand, 2007; C. A. Anderson \& Dill, 2000; Ip, Jacobs, \& Watkins, 2008). Studien zur Computerspielnutzung im Vorschulalter existieren im Gegensatz zum Fernsehen nicht, was vermutlich auf die geringere Verbreitung des Computerspielens in diesem Lebensalter (vgl. Kapitel 1.1.1) zurückzuführen ist.

In der Untersuchung von Sharif und Sargent (Sharif \& Sargent, 2006) zeigte sich für das Computerspielen, dass der Anteil von Schülern mit nach Selbstauskunft unterdurchschnittlichen Leistungen ab einer Stunde Computerspielzeit ansteigt und der Anteil von Schülern mit überdurchschnittlichen Leistungen abfällt. Dabei erweist sich nur die Nutzungszeit an Schultagen, nicht jedoch an Wochenenden als entscheidend. Bei Betrachtung weiterer soziodemographischer, sozioökonomischer, psychometrischer, mediennutzungsbezogener und medienerziehungsbezogener Einflussfaktoren innerhalb einer logistischen Regression verliert jedoch der Prädiktor Spielzeit seine Bedeutung für die Erklärung von Schulleistung. In den anderen Studien bleiben die negativen Zusammenhänge zwischen Computerspielnutzungsparametern und Schulleistung auch unter Kontrolle weiterer Variablen wie Geschlecht (Anand, 2007; Ip, et al., 2008), Geschlecht und aggressionsbezogenen Maßen (C. A. Anderson \& Dill, 2000), Geschlecht, aggressionsbezogenen Maßen und elterlichem Medienerziehungsverhalten (Gentile, et al., 2004) sowie Geschlecht, Migrationsstatus, elterliches Bildungsniveau, elterliche Zuneigung und elterliche Gewalt bzw. in einer Pfadanalyse Geschlecht, elterliche Bildung, elterliches Medienerziehungsverhalten und Sprachfähigkeiten bei der Einschulung (Mößle, et al., 2007) bestehen.

Hinsichtlich der Rolle der genutzten Inhalte kommen die Studien zu uneinheitlichen Befunden. In der Studie von Mößle et al. (2007) zu Grundschulkindern vierter Klassen im Alter von ca. 10 Jahren erwies sich insbesondere die Nutzung gewalthaltiger Spiele und weniger die Nutzungszeit als relevant für Schulnoten in den Fächern Deutsch, Sachkunde und Mathematik, wenn die in der Studie berücksichtigten Kontrollvariablen konstant gehalten wurden. In der Untersuchung von

23 Die Querschnittstudie von Roe, Eggermont und Minnebo (2001) mit einer Stichprobe von rund 1000 Schülern $(M[$ Alter] = 9.5) wurde aus der Zusammenstellung ausgeschlossen, da die Dokumentation der Ergebnisse und des methodischen Vorgehens in der Publikation nicht die wissenschaftlichen Mindeststandards erfüllt. 
Anderson und Dill (2000) an einer weitaus älteren Stichprobe von durchschnittlich 19-jährigen Collegestudenten stellte hingegen nur die Nutzungsdauer, nicht jedoch die Nutzung gewalthaltiger Spiele einen relevanter Prädiktor für Collegenoten dar. Gentile et al. (2004) berücksichtigten in ihrer Befragung 14-jähriger Jugendlicher Nutzungszeiten und Nutzungsinhalte von Computerspielen sowie die Präferenz für gewalthaltige Computerspiele und die Veränderung dieser Präferenz in den letzten Jahren als unabhängige Variablen. In einer Regressionsanalyse erweisen sich unter Berücksichtigung von Kontrollvariablen sowohl die Spielzeit als auch die Nutzung gewalthaltiger Spiele als relevante Prädiktoren für Schulleistung, während in einer Pfadanalyse nur die Nutzungsdauer als relevante Einflussgröße stabil bleibt (Gentile, et al., 2004).

Zwei Studien verzichten vollständig auf die Berücksichtigung von Kontrollvariablen, wodurch die Aussagekraft dieser beiden Studien als deutlich eingeschränkt gelten muss. Die Befragung von Roberts, Foehr und Rideout (2005) im Auftrag der Kaiser Family Foundation kommt zu dem Ergebnis, dass unter den erfassten Medienaktivitäten, darunter auch Fernseh- und DVD-Nutzung, nur die Computerspielzeit signifikant negativ mit Schulnoten korreliert. Schüler, die für sich schlechte Schulnoten als typisch angeben (C, D und schlechter) spielen täglich ca. 20 Minuten länger Videospiele als Schüler mit besseren Noten (Roberts, Foehr \& Rideout, 2005). In der Studie von Chan und Rabowitz finden sich negative Zusammenhänge der Nutzungszeit sowohl von Konsolen- als auch von Onlinespielen ${ }^{24}$ mit der aktuellen Durchschnittsnote, nicht aber mit der der letzten Englischund Mathematiknote (Chan \& Rabinowitz, 2006).

Wie für das Fernsehen wurde auch in Hinblick auf das Computerspielen die These aufgestellt, dass der Zusammenhang mit schulischen Leistungen über eine kurvilineare Beziehung vermittelt sein könnte, indem moderate Nutzer die höchsten, Nichtnutzer und Exzessivnutzer die niedrigste Leistung aufweisen. Die Daten von Anand (2007) deuten auf eine kurvilineare Beziehung hin, jedoch nicht mit einem aktuell erhobenen Leistungsmaß (der aktuellen Durchschnittsnote), sondern den Ergebnissen des SAT am Ende der High-School und hier auch nur für den mathematischen Teil und bei männlichen Befragungsteilnehmern. Bei Sharif und Sargent (2006) steigt der Anteil von Schülern mit unterdurchschnittlichen Leistungen erst ab einer werktäglichen Nutzungszeit von einer Stunde und einer wochenendtäglichen Nutzungszeit von mehr als acht Stunden an. Hier erscheint jedoch, ähnlich wie beim Fernsehen, zusätzliche Forschung nötig, um valide Aussagen zur Art des Zusammenhangs zu ermöglichen.

24 Hinsichtlich dieser Systematisierung der Autoren ist darauf hinzuweisen, dass auch Konsolenspiele inzwischen in hohem Maße online genutzt werden und die Unterscheidung nach Konsolen- und Onlinespielen damit nicht mehr zeitgemäß ist. 
Wenngleich damit die aktuellen Studien zu Computerspielen und Schulleistung relativ einheitlich auf einen negativen Zusammenhang hinweisen, muss die Datenlage insgesamt noch als rudimentär bewertet werden. Da hier im Gegensatz zum Fernsehen keine veröffentlichten Längsschnittdaten ${ }^{25}$ vorliegen, kann insbesondere auf die Wirkrichtung des Zusammenhangs noch nicht zweifelsfrei geschlossen werden. Zwar wurde in vielen Studien der Versuch unternommen, diesen Problemen durch die Kontrolle weiterer Variablen sowie Pfadanalysen zu begegnen. Diese Berechnungen ersetzen jedoch keinen echten Längsschnitt oder experimentelle Interventionsstudien, in welchen das Mediennutzungsverhalten in randomisierten Kontrollgruppendesigns gezielt manipuliert wird. Zudem berücksichtigen einige Studien zwar den sozioökonomischen Status sowie weitere Kontrollvariablen, keine jedoch die Intelligenz der Befragungsteilnehmer, obgleich diese beim Fernsehen als besonders relevanter Moderator diskutiert wird.

\subsubsection{Erklärungshypothesen zum Zusammenhang von Bildschirmmediennutzung und Schulleistung}

"What television can and will achieve occasionally and what it does regularly are not the same" (Comstock \& Scharrer, 1999, S. 231).

Die aktuellen Forschungsdaten der letzten zehn Jahre zeigen auf, dass sowohl eine erhöhte Beschäftigungszeit mit Bildschirmmedien als auch die Nutzung von Unterhaltungs- (Fernsehen) und gewaltbetonten Angeboten (Computerspiele) mit schlechteren Schul- und Studienleistungen in Zusammenhang steht. Gleichfalls wird erkennbar, dass für die Nutzung hochwertiger Edutainmentformate und geringe Nutzungszeiten gegenläufig positive Zusammenhänge mit Schulleistungen berichtet werden.

In der Vergangenheit wurden vielfältige Wirkannahmen zum Zusammenhang von Mediennutzung und Schulleistung postuliert, die sich zunächst grob den sogenannten Stimulierungshypothesen (facilitation hypotheses) und Minderungshypothesen (inhibition hypotheses) zuordnen lassen (vgl. Shin, 2004). Stimulierungshypothesen gehen davon aus, dass Bildschirmmediennutzung das Lernen und die kognitive Entwicklung fördert (stimuliert), indem Medienangebote entweder unmittelbar Wissensbestände und Lernstrategien vermitteln, welche schulische Leistungen von Kindern verbessern können (Ennemoser, 2007; Singer \& Singer, 2001) oder ein Interesse für neue Themen anregen, wodurch eine weiterführende Auseinandersetzung mit neuen Wissensgebieten - zum Beispiel über das Lesen oder

25 In Vorbereitung befindet sich inzwischen eine Publikation erster Ergebnisse der Panelstudie unseres Projektes Mediennutzung und Schulleistung (Mößle, Kleimann, Rehbein, \& Pfeiffer, 2010). 
den Besuch einer Ausstellung - motiviert wird (Comstock \& Scharrer, 1999, S. 230). Befürworter der Stimulierungshypothese beziehen sich in der Regel auf die Nutzung von anregenden oder pädagogisch wertvollen Angeboten und konzentrieren sich auf die Frage, ob bestimmte Bildschirmmedienangebote Lernprozesse anregen, kognitive Fähigkeiten vermitteln oder nutzbar gemacht werden können, um vorhandene Lernprozesse zu optimieren.

Minderungshypothesen stützen sich hingegen auf die Annahme einer nachteiligen Wirkung von Bildschirmmediennutzung auf Lern- und Leistungsergebnisse und postulieren, dass höherwertige kognitive Verarbeitungsvorgänge durch diese Angebote nicht effektiv gefördert oder sogar in Mitleidenschaft gezogen werden, Zeit von realweltlichen Lernerfahrungen abgezogen wird oder insgesamt ein Verhalten begünstigt wird, welches schulischen Lernerfolgen entgegensteht. Minderungshypothesen beziehen sich maßgeblich auf die Annahme, dass sich Bildschirmmedien trotz darin möglicherweise enthaltener positiver Potentiale insgesamt nachteilig auf schulische Leistungen auswirken, da positive Wirkpotentiale die Alltagsmediennutzung der meisten Rezipienten nicht dominieren:

"Our reasoning is that the entertainment predominantly viewed is not designed to be pedagogic and so lacks the repetition, focus, and order of the instructional examples that have proven effective" (Comstock \& Scharrer, 1999, S. 231).

Dabei stehen zwei Gesichtspunkte im Vordergrund: Die hohe zeitliche Beanspruchung durch Bildschirmmedien im Freizeitmenu und die Dominanz von Unterhaltungsformaten sowie gewalthaltigen Angeboten im Medienmenu von Kindern und Jugendlichen (vgl. Kapitel 1.1.2).

Von besonderem Interesse für diese Untersuchung ist die Frage, welche Bedeutung der im Medienmenu junger Menschen dominierenden Unterhaltungsmediennutzung und der gerade bei männlichen Nutzern zu beobachtenden Präferenz für gewalthaltige Angebote zukommt. Auf Basis der dargestellten Befundlage zum Zusammenhang von Mediennutzung und Schulleistung sowie der Notwendigkeit einer thematischen Eingrenzung wird deshalb ein besonderer Schwerpunkt auf die Frage gelegt, welcher Erklärungswert Minderungshypothesen für den $\mathrm{Zu}$ sammenhang von Bildschirmmediennutzung und Schulleistung zukommen kann, die im Folgenden kurz dargestellt werden sollen.

Die Zeitverdrängungshypothese kann als besonders bekannte Minderungshypothese zum Zusammenhang von Mediennutzung und Schulleistung gelten (Ennemoser \& Schneider, 2007, S. 349). Sie postuliert, dass dem Lernen unmittelbar zugutekommende Aktivitäten wie Lesen und Hausaufgaben machen, Schlaf- und Erholungszeiten, aber auch andere Freizeitbeschäftigungsweisen wie zum Beispiel körperliche Betätigung oder soziale Aktivitäten zugunsten der Bildschirmmediennutzung reduziert werden und in der Folge schulische Leistungen in Mitleidenschaft gezogen werden (Cummings \& Vandewater, 2007; Gentile, et al., 2004; 
Mößle, et al., 2007; Williams, et al., 1982, S. 35 ff.). Auch die Annahme einer Qualitätsminderung des Lernens durch eine parallel betriebene Medienrezeption wird vielfach unter der Zeitverdrängungshypothese subsummiert und zum Zwecke einer präziseren Terminologie auch als qualitative Zeitverdrängung bezeichnet (Beentjes \& Van der Voort, 1988; Gaddy, 1986, S. 346). ${ }^{26}$

Die Aggressionshypothese (auch Inhaltshypothese) ${ }^{27}$ postuliert in Folge der Nutzung gewalthaltiger Medien ein problematisches Sozialverhalten in Form erhöhter Aggressivität oder Impulsivität und damit eine Erhöhung der Wahrscheinlichkeit für Verhaltensweisen, die schulischen Erfolgen entgegenstehen (D. R. Anderson, et al., 2001, S. 39; Comstock \& Scharrer, 1999, S. 236).

"Aggressive behavior can lead to spirals of lower output, lower quality of work, poorer grades, reduced motivation to succeed, and a negative cycle of academic failure and alienation from the culture of achievement and the goals represented by the educational system" (D. R. Anderson, et al., 2001, S. 39, zitiert nach Williams, 1986; MacBeth, 1996).

Damit knüpft die Aggressionshypothese unmittelbar an die Forschung zum Zusammenhang von Gewaltmedienrezeption und Aggressivität an, aus der sie auch ihre maßgebliche Legitimation erhält.

Nach Comstock und Scharrer lässt sich eine dritte heterogene Kategorie von Minderungshypothesen als Interferenzhypothesen einordnen (Comstock \& Scharrer, 1999, S. 255 ff.). Interferenzhypothesen postulieren in Folge der Mediennutzung motivationale Veränderungen oder mentale Leistungseinbußen, die sich nachteilig auf die Schulleistungsfähigkeit auswirken und stützen sich dabei auf bestimmte Präsentationsformate in den Medien. Wie die meisten Minderungshypothesen zum Zusammenhang von Bildschirmmediennutzung und Schulleistung sind auch die Interferenzhypothesen maßgeblich auf Grundlage der Fernsehwirkungsforschung entstanden.

Insgesamt lassen sich der Literatur vier besonders prominente Annahmen entnehmen, die sich den Interferenzhypothesen zuordnen lassen. Die Passivitätshypothese (Passivity Hypothesis) postuliert, dass der im Vergleich zum Lesen geringere mentale Aufwand, der für die Nutzung bildschirmgestützter Unterhaltungsmedien, insbesondere dem Fernsehen notwendig ist, als passive Haltung auf das Lesen übertragen wird und damit die Lesekompetenzentwicklung beeinträch-

26 Unter der Zeitverdrängungshypothese wird inzwischen auch eine psychische Abhängigkeit von Medien (insbesondere Computerspielen) diskutiert, die ebenfalls schlechtere Schulleistungen in Zusammenhang mit Mediennutzung erklären könnte (Anand, 2007, S. 552; Baier \& Rehbein, 2009; Mößle, et al., 2007; Rehbein, Kleimann, \& Mößle, 2009 b).

27 Aufgrund der engeren Ausrichtung dieser Hypothese auf gewalthaltige Medien wird die Aggressionshypothese auch unter dem Label "Inhaltshypothese" geführt, eine Terminologie, die jedoch keine eindeutige Abgrenzung zu einigen Kognitionshypothesen erlaubt, da sich diese zum Teil ebenfalls auf bestimmte inhaltliche Merkmale von Medien stützen. 
tigen kann (Ennemoser, 2007; Koolstra, et al., 1997; Shin, 2004). In ähnlicher Weise postuliert die Lesebeeinträchtigungshypothese (Reading Depreciation Hypothesis), dass unterhaltsame Mediennutzungserfahrungen die Motivation zur Beschäftigung mit vergleichsweise mental anstrengenderen Lernkontexten und dem Lesen reduzieren (Ennemoser \& Schneider, 2007, S. 349) und damit sowohl Einstellungen zum Lesen als auch schulische Leistungen negativ beeinflussen (Ennemoser, 2007; Koolstra, et al., 1997).

Zwei weitere Hypothesen postulieren in Folge von Mediennutzung die unmittelbare Beeinträchtigung von kognitiven Informationsverarbeitungsprozessen. Die Konzentrations-Defizit-Hypothese (Concentration Deterioration Hypothesis) postuliert, dass die im Rahmen einer Bildschirmmedienrezeption erfolgende Überstimulierung mit Bildreizen die Konzentrationsfähigkeit herabsetzt (Christakis, Zimmerman, DiGiuseppe, \& McCarty, 2004; Ennemoser, 2007) bzw. eine oberflächliche Weiterverarbeitung von Informationen anregen kann (Shin, 2004). Als mögliche Ursache wird angeführt, dass Mediennutzung eher eine fixierte Aufmerksamkeitszuwendung auf einen sich ständig verändernden Stimulus nahelegt und weniger selbstkontrollierte, selektive Aufmerksamkeitszuwendung zu einer bestimmten Aufgabe fördert (Ennemoser \& Schneider, 2007, S. 349; Zimmerman $\&$ Christakis, 2007, S. 987). Hieraus wird abgeleitet, dass das Erlernen neuer Informationen innerhalb einer von Bildschirmmedien geprägten starken Reizumgebung mit geringen Ansprüchen an die mentale Selbstdisziplin zu einer Verminderung der Zuwendungsfähigkeit zu Lernreizen mit geringerer Reizintensität in der Schule führen könnte. Insbesondere für die frühkindliche Mediennutzung wurde in diesem Zusammenhang befürchtet, dass sie kognitive Verarbeitungsprozesse und die neurologische Entwicklung in einer Weise beeinflussen könnte, dass hier die befürchteten Aufmerksamkeitseinbußen besonders wahrscheinlich werden (vgl. Christakis, et al., 2004; Stevens \& Mulsow, 2006; Zimmerman \& Christakis, 2007).

"Sensory stimulation within reasonable limits certainly has a beneficial effect on muscle tone and alertness. A sensory assault, however, heightens tension to levels of discomfiture and also hobbles the capacity for maintaining attention. If the organism is immature, there is greater danger that the impedance of perceptual readiness (the substrate of attention) can lead to an unsteady developmental process. Sesame Street's use of intense visual and auditory patterns to capture attention, its rapid perceptual shifts through the use of zoom lenses and quick dissolves, and its studied avoidance of time lags between messages strongly interfere with the emergence of the perceptual readiness state" (Halpern, 1975, S. 69).

Eine neuere kognitive Interferenzhypothese ist die Gedächtnis-Defizit-Hypothese (auch Löschungshypothese, Deletion Hypothesis). Dieser Annahme zufolge 
kann die Dekodierung medialer Informationen aufgrund der damit verbundenen Erregungsvorgänge kognitive Ressourcen von anderen unvollendeten Lern- und Verarbeitungsvorgängen abziehen bzw. binden. Diese Annahme stützt sich insbesondere auf gewalthaltige Medien, da von diesen vermutet wird, dass sie in besonderer Weise Arousal- und Stressreaktionen auslösen können. Die Hypothese knüpft damit an psychologische Erkenntnisse zur Beeinflussbarkeit von Lern- und Informationsverarbeitungs-prozessen durch emotionale Erlebniszustände an. Die Hypothese wurde insbesondere von Hirnforschern wie Spitzer (2005) und Scheich (2006) vertreten:

„Es ist offensichtlich, dass Medienkonsum mit stark emotional wirksamen Inhalten eine bevorzugte Verankerung solcher Informationen bewirkt. Dies geschieht in Konkurrenz zu weniger aufwühlenden Erfahrungen z. B. im Schulalltag. (...) Die Verankerung einer Information im Langzeitgedächtnis dauert mehr als 24 Stunden. Wird während dieser Zeit die Information wiederholt oder durch ähnliche Informationen variiert, verstärkt sich die Verankerung im Langzeitgedächtnis. Dies erklärt einerseits den massiven Effekt von regelmäBigem Langzeitkonsum bestimmter Medien, andererseits aber auch den Sinn nachmittäglicher Hausaufgaben in der Schule oder Ganztagsschule mit Stoffvertiefungen am Nachmittag. Nimmt TV-Konsum oder Computerspielen die zeitlich und emotional dominierende Rolle im Tagesverlauf ein, hat Schulstoff keine Chance fest im Langzeitgedächtnis verankert zu werden" (Scheich, 2006, S. $227 \mathrm{ff}$.).

\subsubsection{Einordnung der vorliegenden Arbeit in die bestehende Erkenntnislage}

"Although we were able to show reliable and consistent negative effects of heavy TV viewing on reading achievement, the underlying inhibitory processes are still unclear. (...) Apparently, several inhibition processes make a difference to some extent, and they may interact and accumulate in producing negative effects on academic performance over time" (Ennemoser \& Schneider, 2007, S. 365).

Während die Minderungshypothesen bislang in Bezug auf das Computerspielen kaum systematisch und vor dem Hintergrund ihrer Bedeutung für die schulische Leistungsentwicklung diskutiert und untersucht wurden, haben einige Thesen hinsichtlich des Fernsehens eine partielle empirische Bestätigung erfahren. So konnte etwa bezüglich der Zeitverdrängungshypothese gezeigt werden, dass erhöhte Mediennutzung mit geringerer Lese- und Lernzeit (Cummings \& Vandewater, 2007) sowie geringeren Schlafzeiten in Zusammenhang steht (Crönlein, et al., 2007; Heins, et al., 2007; Tazawa \& Okada, 2001; van den Bulck, 2004 b). Auch hin- 
sichtlich der längsschnittlichen Bedeutung des Mediators "Zeitverdrängung" existieren bestätigende (J. G. Johnson, et al., 2007; Koolstra \& Van der Voort, 1996; Koolstra, et al., 1997), zum Teil jedoch auch gemischte Befunde (Ennemoser \& Schneider, 2007). Auch die Pfadanalysen von Shin (2004) konnten die Relevanz des Mediators Zeitverdrängung für den negativen Zusammenhang von Fernsehnutzung und Schulleistung bestätigen. Insgesamt ist jedoch in nahezu allen Untersuchungen nicht zweifelsfrei klar, ob wirklich Zeitverdrängungsmechanismen und nicht anderweitige, zum Beispiel motivationale Veränderungen, erfasst werden. So stellt sich zum Beispiel die Frage, ob Defizite in der Erledigung von Hausaufgaben in jedem Fall auf Zeitverdrängungsprozesse zurückzuführen sind und als Stärkung dieser These gewertet werden können (vgl. J. G. Johnson, et al., 2007; Shin, 2004).

Unter den Kognitionshypothesen hat die Konzentrations-Defizit-Hypothese trotz im Vergleich zur Zeitverdrängungshypothese geringen Forschungsanstrengungen (vgl. Ennemoser \& Schneider, 2007, S. 349) partielle Bestätigung im Rahmen von Längsschnitt- (J. G. Johnson, et al., 2007; Koolstra \& Van der Voort, 1996) und Querschnittstudien (vgl. Shin, 2004) erfahren. Zudem konnten Zusammenhänge zwischen frühkindlicher Fernsehnutzung und späteren Symptomen einer Aufmerksamkeitsstörung beobachtet werden (Christakis, et al., 2004; Hancox, et al., 2005). Gerade hinsichtlich der Konzentrations-Defizit-Hypothese ist jedoch bislang eine plausible theoretische Einbettung der heterogenen Daten zu vermissen. Zudem bestehen zum Teil auch abweichende Befundmuster, indem zum Beispiel in der Längsschnittstudie von Ennemoser und Schneider keine Zusammenhänge zwischen Fernsehnutzung und Aufmerksamkeitsleistung ${ }^{28}$ aufgefunden werden konnten (Ennemoser \& Schneider, 2007). Die Bedeutung der anderen Kognitionshypothesen ist auf Basis der empirischen Datenlage sehr schwer zu beurteilen. Bei Ennemoser und Schneider ergab sich keine Bewährung der Lesebeeinträchtigungshypothese, indem zwar ein negativer Einfluss von Fernsehzeit auf den Mediator Lesezeit nachgewiesen werden konnte, jedoch kein Einfluss der Lesezeit auf die Lesefähigkeit. Auch Leseeinstellungen wurden nicht durch das Fernsehen, sondern vielmehr durch geringe Lesefertigkeiten negativ beeinflusst (Ennemoser \& Schneider, 2007). Die Gedächtnis-Defizit-Hypothese wurde bislang im Rahmen von empirischen Untersuchungen zum Zusammenhang von Mediennutzung und Schulleistung noch nicht berücksichtigt und auch anderweitig, wie noch aufzuzeigen sein wird, kaum untersucht. Maßgebliche Stützung erfährt die Gedächtnis-Defizit-Hypothese bislang in erster Linie aus Werbewirkungs- und Nachrichtenwirkungsstudien, in denen gezeigt werden konnte, dass Werbefilme im Kontext von gewalthaltigen Filmen schlechter erinnert werden als im Kontext von gewaltneutralen Filmen (Bushman \& Bonacci, 2002) und Erinnerungen an die Details von Nach-

28 Die Forschungsgruppe setzte für die Operationalisierung von Konzentrationsleistung einen Aufmerksamkeitstest ein. 
richtenformaten im Falle expliziter Gewaltdarstellungen innerhalb der Formate geringer ausfallen (Lang, Newhagen, \& Reeves, 1996; Newhagen \& Reeves, 1992). Lang und Kollegen stützen sich dabei auf die Annahme, dass schockierende gewalthaltige Bilder in erhöhter Weise Informationsverarbeitungskapazitäten binden:

„Following this reasoning it is likely that negative video results in a call for additional resources at both the encoding and storage level of processing. This may result in a temporary overload of the processing system and is likely to have detrimental effects on both the encoding and storage of the message" (Lang, et al., 1996, S. 464).

In diesen Untersuchungen sind jedoch die Lerninhalte zugleich Medieninhalte, weshalb eine unmittelbare Übertragung dieser Daten auf schulische Lernprozesse unzulässig erscheint.

In ähnlicher Weise lückenhafte Erkenntnisse bestehen in Hinblick auf die Aggressionshypothese, die in den meisten Studien nicht Gegenstand der überprüften Modellannahmen ist (vgl. Ennemoser \& Schneider, 2007; Shin, 2004). Dies ist sicherlich auch der Tatsache geschuldet, dass die differenzielle Bedeutung der Nutzung gewalthaltiger Fernsehformate für schulische Leistungen bislang kaum untersucht wurde (mit Ausnahme von D. R. Anderson, et al., 2001; Mößle, et al., 2007). Gentile und Kollegen (2004) fanden jedoch im Rahmen von Kausalanalysen eine Bestätigung für die Relevanz des Mediators Aggressivität in Hinblick auf gewalthaltige Computerspiele, indem deren Nutzung über eine erhöhte Feindseligkeit schlechtere Schulleistungen vorhersagte. Zudem besteht inzwischen eine recht solide Forschungsbasis zu der Frage, wie sich die Nutzung gewalthaltiger Medien auf aggressionsnahe Konstrukte auswirkt (vgl. Kapitel 1.3.3).

Somit sind trotz langjährigen Forschungsbemühungen nach wie vor gesicherte Annahmen über das Wirkungsgefüge zwischen den beobachteten Phänomenen und über den Erklärungswert der postulierten Annahmen nicht möglich (vgl. D. R. Anderson, et al., 2001, S. 2; Ennemoser \& Schneider, 2007, S. 365; Gaddy, 1986, S. 341; Mößle, et al., 2007; Williams, et al., 1982, S. 35 ff.). Das nachfolgende Diagramm veranschaulicht diese Situation und greift die in der Forschung häufig diskutierten Erklärungsansätze in einem hypothetischen Arbeitsmodell auf (vgl. Abbildung 8). 


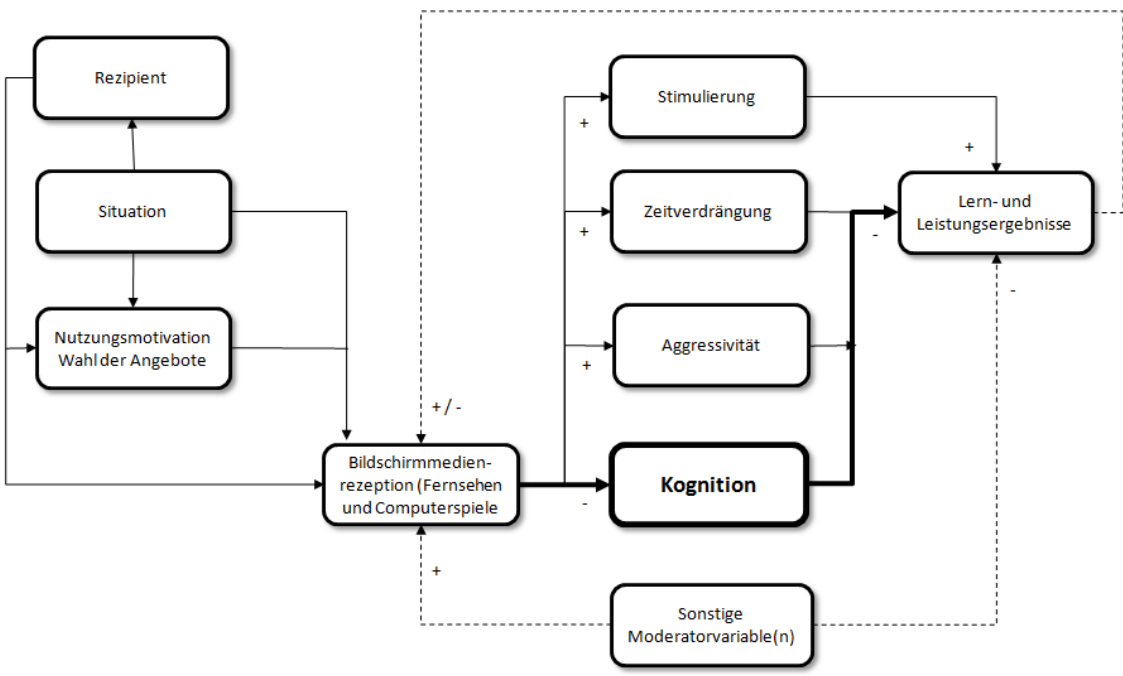

Abbildung 8. Hypothetisches Arbeitsmodell zum Zusammenhang von Bildschirmmediennutzung und Lern- und Leistungsergebnissen (basierend auf einer Zusammenfassung des Forschungsstandes und unter Rückgriff auf zentrale Minderungshypothesen)

Das Modell berücksichtigt zunächst die Grundannahme, dass spezifische Charakteristika in der Person des Rezipienten sowie situative Lebensumstände die Art und Weise, wie sich Menschen mit Bildschirmmedien beschäftigen, maßgeblich determinieren (vgl. D. R. Anderson, et al., 2001). Diese Annahme knüpft an den medienwissenschaftlichen Konsens an, dass Rezeptionsprozesse nicht alleine vom genutzten Medium bestimmt werden, sondern auch in Abhängigkeit von der Person des Rezipienten stattfinden, der die Angebote auswählt und dessen Persönlichkeit das Rezeptionsgeschehen bestimmt (vgl. Schmitt, 2004). Interindividuelle Unterschiede in diesem Setting könnten demnach einen Einfluss darauf haben, inwieweit sich Wirkungen auf die Leistungsebene entfalten.

Grundsätzlich ist es wahrscheinlich, dass der Zusammenhang zu einem gewissen Anteil über Moderatorvariablen erklärt werden kann, die auf ähnliche Weise sowohl die Nutzung von Bildschirmmedien als auch die Schulleistung beeinflussen (vgl. Abbildung 8, gestricheltes System unten). Obgleich inzwischen einige Studien eine Vielzahl von Moderatoren kontrollieren, kann aufgrund der Komplexität und multikausalen Genese schulischer Leistungen (vgl. Kapitel 1.2.1) nicht ausgeschlossen werden, dass in den Studien bislang nicht erfasste, konfundierende Variablen für Teile des Zusammenhangs zwischen Medienzeit und Schulleistung 
verantwortlich sind (vgl. Sharif \& Sargent, 2006, S. 1069). Auch ist denkbar, dass negative Schulleistungen unmittelbar auf die Nutzungsintensität von Bildschirmmedien zurückwirken könnten, indem zum Beispiel ein Leistungsabfall in der Schule zu einer Suche nach alternativen Belohnungsmöglichkeiten und einer erhöhten Attraktivität von Bildschirmmedien für den betreffenden Schüler führt (vgl. Willoughby, 2008, S. 202). Hier sind auch negative Feedbackprozesse denkbar, indem bei Leistungseinbußen in der Schule das Mediennutzungsverhalten zugunsten vermehrter Lernanstrengungen reguliert wird (vgl. Abbildung 8, gestricheltes System oben).

Im Kern des Modells steht jedoch die mit dem Forschungsstand insgesamt gut zu vereinbarende Annahme, dass durch die Nutzung von Bildschirmmedien Veränderungsprozesse in der Person des Rezipienten oder seiner Alltagsgestaltung ausgelöst werden könnten, die schulische Leistungen unmittelbar beeinflussen können (vgl. Abbildung 8, mittleres hervorgehobenes System). Hier werden die bereits diskutierten Wirkhypothesen aufgegriffen. Wie bereits ausgeführt, kann bislang jedoch keine verlässliche Aussage darüber gemacht werden, wie hoch der Erklärungswert der aufgeführten hypothetischen Wirkmechanismen und der damit angesprochenen Mediatoren einzuschätzen ist

Ziel des interdisziplinären Forschungsprojektes "Mediennutzung und Schulleistung" des Kriminologischen Forschungsinstituts Niedersachsen ist es, an dieser Forschungslücke ansetzen. Ein erster Kernbestandteil des Projektes ist eine medienwissenschaftliche Längsschnittstudie, in der Grundschüler von der 3. bis 6 . Klasse begleitet werden, und ihr Mediennutzungsverhalten sowie ihre Sozial-, Intelligenz- und körperliche sowie schulische Entwicklung jährlich erfasst wird (Mößle, et al., 2010). Diese Studie sieht zudem für einen Teil der Schüler medienpädagogische Interventionseinheiten vor, deren Wirksamkeit im Vergleich zu den übrigen Kindern (Kontrollgruppe, Baselinegruppe) ebenfalls überprüft wird (Kleimann \& Mößle, 2008). Während in der Längsschnittstudie langfristige Wechselwirkungen zwischen Mediennutzungs- und Entwicklungsparametern analysiert werden sollen, dient die vorliegende experimentelle Studie dem Ziel kurzfristige Effekte der Nutzung von Bildschirmmedien auf komplexe kognitive Leistungsaspekte zu untersuchen, die eine hohe Relevanz für schulische Leistungen aufweisen. Experimentelle Studien können in besonderer Weise dazu beitragen, kausale Wirkannahmen zu lernbezogenen Primärwirkungen von Bildschirmmedien zu überprüfen und damit der Vielfalt konkurrierender und in der Feldforschung nur schwer zu untersuchender Hypothesen sowie gesellschaftlich verbreiteten Alltagsbefürchtungen eine pragmatische empirische Befundlage gegenüberzustellen. Da hierbei eine Untersuchung der Wirkungen problematischer Formate im Vordergrund stehen soll, konzentriert sich diese Arbeit auf gewalthaltige Bildschirmmedien am Beispiel der hinsichtlich ihrer Relevanz für schulische und kognitive Leistungen 
bislang wenig untersuchten gewalthaltigen Computerspiele. Damit ist es Anliegen dieser Arbeit, den Erklärungswert zentraler kognitiver und affektiver Minderungshypothesen für den aufgefundenen negativen Zusammenhang zwischen Unterhaltungsmediennutzung und Schulleistung am Beispiel der Nutzung gewalthaltiger Computerspiele auf ihre Relevanz hin zu überprüfen und zur Theoriebildung in diesem Feld beizutragen.

Im folgenden Kapitel wird der Frage nachgegangen werden, welche Kenntnisse bislang zu den kognitiven und affektiven Wirkungen von gewalthaltigen Computerspielen vorliegen.

\subsection{Gewalthaltige Computerspiele: Kognitives und emotionales Wirkpotential}

\subsubsection{Computerspielen und kognitive Beanspruchung}

Computerspielen unterscheidet sich hinsichtlich der beteiligten Sinnesmodalitäten kaum vom klassischen Fernsehen: auch hier werden akustische und visuelle Informationen über technische Ausgabemedien vermittelt und bestimmen auf diese Weise das Rezeptionsgeschehen. Hinsichtlich der beanspruchten kognitiven Leistungen bestehen dennoch deutliche Unterschiede: Als interaktives Medium wird das Rezeptionsgeschehen beim Computerspiel durch die Eingaben des Nutzers und die Folgen dieser Eingaben im Spiel bestimmt, die einen wechselseitig-reaktiven Kreislauf bilden (vgl. Klimmt, 2004, S. 3). Computerspiele erfordern deshalb im Vergleich zu Filmen, die auch neben anderen Aktivitäten unter geringer aktiver Zuwendung genutzt werden können, ein beständiges Maß an Aufmerksamkeit (Kunczik \& Zipfel, 2004, S. 201).

Dabei kommt es auf das verwendete Computerspiel an, welche Leistungen die Spieler erbringen müssen, um die spielimmanenten Ziele zu erreichen. Der Computerspielforscher Klimmt (2004, S. 5) schlägt vor, Computerspiele insbesondere danach zu unterscheiden, ob sie eher Komplexität oder eher Geschwindigkeit in den Vordergrund stellen: Spiele, die eher Komplexität in den Vordergrund stellen, erfordern vom Spieler statt schneller Spielhandlungen vorzugsweise die Berücksichtigung und Überwachung einer Vielzahl von sich fortlaufend verändernden Informationen und die Umsetzung dieser Informationen in strategische Überlegungen. Typische Beispiele für derartige Spiele sind Denkspiele, Strategiespiele und Wirtschafts- bzw. Aufbausimulationen. Stattdessen legen Spiele, die eher Spielgeschwindigkeit in den Vordergrund stellen, den Akzent auf schnelle Reaktionen, die Erfassung und geschickte Ausnutzung räumlich-visueller Zusammenhänge und die schnelle Koordination komplexer Steuerbefehle. Gewalthaltige 